\title{
RESCHEDULING METHODS FOR MANUFACTURING FIRMS APPLYING MAKE-TO-ORDER STRATEGY
}

\author{
Song, L. J.; Gu, H. P.; Jin, S. Y. \& Zhao, H. \\ College of Mechanical Engineering, Chongqing University of Technology, Chongqing 400054, China \\ E-Mail: sglijn@163.com
}

\begin{abstract}
To combat the problem of frequent production plan change in an environment where manufacturing systems are constantly varying, an improved hybrid rescheduling strategy is proposed. Disturbance factors are classified according to their origin and influence, and their disturbance degree is determined using a fuzzy-neural network. On the basis of this, an adaptive rescheduling process for manufacturing systems has been constructed and an improved hybrid rescheduling strategy is proposed. Finally, the effectiveness of this improved strategy is illustrated by a case study of practical manufacturing. The results show that this strategy can effectively reduce rescheduling times and maintain the stability of manufacturing systems.

(Received, processed and accepted by the Chinese Representative Office.)
\end{abstract}

Key Words: $\quad$ Manufacturing Systems, Disturbance Degree, Rescheduling, Fuzzy-Neural Network

\section{INTRODUCTION}

Faced with competitive markets, an ever-changing and complex manufacturing environment, and customers' requirements for personalized, small-patch, low-cost and high-quality products, a number of manufacturing firms are shifting their manufacturing mode from Maketo-Stock (MTS) to Make-to-Order (MTO) [1, 2]. For manufacturing firms adopting an MTO production mode, rescheduling management is an effective method to decide on market strategy and production, from the perspective of both the internal and external environments. It is similarly effective when aiming to integrate warehousing logistics, production and management, and to deliver personalized products to customers on schedule and in the right quantity [3]. Current research on job shop scheduling is associated with the proposition that the circumstances are never changed, and that the rescheduling plan, once made, will be carried out until the end. However, the ever-varying market trend and the complicated producing systems of actual production always lead to the occurrence of a disturbance factor. The original scheduling plan therefore inevitably becomes unsuitable, which necessitates an adjustment (i.e. rescheduling). Rescheduling can effectively eliminate the negative effect of changes in firms' external and internal environment on its production system. Thus rescheduling has become one focus of current research into scheduling, and a subject of global interest.

At present, research into rescheduling emphasizes four aspects: rescheduling factors, rescheduling strategy, rescheduling methods and rescheduling performance evaluation [4-7]. More specifically, Chen and Ji [8] proposed a periodic rescheduling strategy with a fixed interval. This solved an improved dynamic schedule model by using a combination of a genetic algorithm and a rolling time domain optimization strategy. The strategy reduced the idle time of the machine, increased the on-time delivery rate, and improved the effectiveness and stability of the schedule model. Pfund [9] used the Lagrange relaxation algorithm to generate a rescheduling plan for a drilling process under a printed circuit board environment with two disturbance factors: uncertain processing time and machine breakdown. Guo and Nonaka [10] explored reschedule strategy and optimization under an environment solely suffering from equipment failure. Akturk and Gorgulu [11] studied equipment failure and 
used the branch and bound method to determine matching dots. They proposed a match-up rescheduling method based on feedback mechanisms, which can ensure the stability of rescheduling. Li et al. [12] proposed a failure-driven rescheduling method to enhance system stability, in which the task was adjusted according to failure prediction. Mendez and Cerda [13] considered task rescheduling caused by unexpected equipment breakdown and maintenance. They were able to use the Mix integer programming method to solve rescheduling problems of parallel batch processing machines. Church and Uzsoy [14] used a hybrid rescheduling strategy (a combination of periodic and event-driven strategy) to solve the unknown reaching time of workpieces in a dynamic environment, with single machines and parallel machines as the object of study. Rangsaritratsamee et al. [15] proposed a hybrid rescheduling strategy to overcome the shortcoming of the periodic rescheduling strategy, where changes in the production system were not being tracked on time. With their new strategy, at each moment of requesting rescheduling, a genetic algorithm was used to optimize the local system so as to maintain system stability. Liu [16] studied the rescheduling of job shops suffering from equipment failure. He used a rolling time domain optimization strategy and designed an improved mechanism of the hybrid rescheduling strategy. Using an improved ant colony system algorithm then allowed him to solve his case, in order to verify the validity.

The above literature demonstrates a number of points. Firstly, the current hybrid rescheduling strategy is a combination of a periodic rescheduling strategy and an event-driven rescheduling strategy. This means that regular rescheduling is conducted, according to the specified cycle, and the effect of unexpected events on the production system is considered. This strategy can make a better response to changes taking place in the production process and can control the amount of calculation. However, only a simple combination of a periodic rescheduling strategy and an event-driven rescheduling strategy has been studied. Furthermore, the effect on production systems of unexpected disturbance factors has been mainly considered, with less attention given to the effect of gradual disturbance factors. There are three shortcomings of this approach.

(1) There has been no comprehensive study on disturbance factors that affect the normal operation of production systems. The disturbance factors studied in current research into rescheduling are: failure of the processing machine; processing time offset of workpieces; and customers' inserted orders. However, the origins of these factors and their influence on production systems are seldom explored profoundly or thoroughly. In actual production, these factors are usually generated at the same time, but exert different effects on production systems.

(2) There has been no comprehensive study on gradual disturbance factors. If production systems do not adequately respond to these factors, rescheduling frequency will increase; leading not only to system oscillation, but also increased computation effort.

(3) The problem as to how to coordinate a periodic rescheduling strategy and an eventdriven rescheduling strategy has not been solved, which will increase rescheduling frequency.

In this paper, the disturbance factors first classified are those which commonly occur in production process of firms adopting an MTO mode. Next, disturbance factors with fuzzy characteristics are thoroughly studied. By introducing the fuzzy neural network technique, a hybrid rescheduling strategy with the minimum interval is proposed. Finally, an adaptive rescheduling process is established, the related cases are simulated, and the validity is verified.

\section{DESCRIPTION AND CLASSIFICATION OF DISTURBANCE FACTORS OF JOB SHOPS}

In actual production, there are always various disturbance factors, which affect the operation of the production system and make the original scheduling plan unsuitable. 
Table I: Classification of disturbance factors of job shops.

\begin{tabular}{|c|c|c|c|c|}
\hline $\begin{array}{l}\text { Origin of } \\
\text { disturbance } \\
\text { factors }\end{array}$ & $\begin{array}{l}\text { Disturbance } \\
\text { factors }\end{array}$ & Effect on production process & $\begin{array}{l}\text { Disturbance } \\
\text { methods }\end{array}$ & $\begin{array}{l}\text { Disturbance } \\
\text { degree }\end{array}$ \\
\hline $\begin{array}{l}\text { Inherent } \\
\text { disturbance } \\
\text { factors } \\
\end{array}$ & $\begin{array}{l}\text { Processing } \\
\text { time offset }\end{array}$ & $\begin{array}{l}\text { Reduces the feasibility and } \\
\text { effectiveness of the original } \\
\text { scheduling plan, and (accumulated } \\
\text { offset) results in delay-shipping }\end{array}$ & Type C & medium \\
\hline \multirow{8}{*}{$\begin{array}{l}\text { Disturbance } \\
\text { factors during } \\
\text { production }\end{array}$} & $\begin{array}{l}\text { Equipment } \\
\text { failure/reboot }\end{array}$ & $\begin{array}{l}\text { Increases or decreases the number } \\
\text { of pieces of equipment, and affects } \\
\text { the workpiece involved }\end{array}$ & Type B & fuzzy \\
\hline & $\begin{array}{l}\text { The decrease } \\
\text { of capacity } \\
\text { utilization }\end{array}$ & $\begin{array}{l}\text { Accumulated disturbance affects } \\
\text { execution of the production plan } \\
\text { and production quality }\end{array}$ & Type C & medium \\
\hline & $\begin{array}{l}\text { Workpiece } \\
\text { reworking }\end{array}$ & $\begin{array}{l}\text { Increases the amount of processing } \\
\text { task in corresponding equipment } \\
\text { and increases WIP }\end{array}$ & Type C & medium \\
\hline & $\begin{array}{l}\text { Workpiece } \\
\text { scrap }\end{array}$ & $\begin{array}{l}\text { If there are few scrap workpieces } \\
\text { and less effect on the scheduling } \\
\text { plan, the original plan can be carried } \\
\text { out; otherwise, the original plan will } \\
\text { be changed }\end{array}$ & Type B & fuzzy \\
\hline & $\begin{array}{l}\text { Change in } \\
\text { WIP }\end{array}$ & $\begin{array}{l}\text { If there is only a small change, and } \\
\text { less effect on the scheduling plan, } \\
\text { the original plan can be carried out; } \\
\text { otherwise, the original plan will be } \\
\text { changed }\end{array}$ & Type B & fuzzy \\
\hline & $\begin{array}{l}\text { Temporary } \\
\text { process } \\
\text { change }\end{array}$ & $\begin{array}{l}\text { Changes the process route of the } \\
\text { current batch, changes the } \\
\text { processing task in the involved } \\
\text { equipment }\end{array}$ & Type C & medium \\
\hline & $\begin{array}{l}\text { Temporary } \\
\text { production } \\
\text { plan change }\end{array}$ & $\begin{array}{l}\text { Decomposes and recombines the } \\
\text { present task }\end{array}$ & Type A & serious \\
\hline & $\begin{array}{l}\text { Flow of } \\
\text { material not } \\
\text { smooth }\end{array}$ & $\begin{array}{l}\text { If there is little accumulated } \\
\text { material, this will only affect the } \\
\text { processing task of the current batch; } \\
\text { if there is much, it will affect the } \\
\text { processing task of all batches }\end{array}$ & Type B & fuzzy \\
\hline \multirow{4}{*}{$\begin{array}{c}\text { Disturbance } \\
\text { factors caused } \\
\text { by change of } \\
\text { an enterprise's } \\
\text { external } \\
\text { environment }\end{array}$} & $\begin{array}{c}\text { Customers' } \\
\text { inserted order }\end{array}$ & $\begin{array}{l}\text { Adds rush workpiece; making the } \\
\text { current scheduling plan invalid }\end{array}$ & Type A & serious \\
\hline & $\begin{array}{l}\text { Reduction or } \\
\text { cancel of } \\
\text { order }\end{array}$ & $\begin{array}{l}\text { Makes the original scheduling plan } \\
\text { invalid, and necessitates a thorough } \\
\text { adjustment to the original plan }\end{array}$ & Type A & serious \\
\hline & Rush order & $\begin{array}{l}\text { Changes workpiece processing } \\
\text { priority, increases rush order, } \\
\text { changes sequence of tasks of the } \\
\text { current batch }\end{array}$ & Type A & serious \\
\hline & $\begin{array}{c}\text { Customers' } \\
\text { requirement } \\
\text { for specific } \\
\text { process }\end{array}$ & $\begin{array}{l}\text { Changes the route of processing the } \\
\text { current workpiece, changes the } \\
\text { processing task in the involved } \\
\text { equipment }\end{array}$ & Type C & medium \\
\hline \multirow{3}{*}{$\begin{array}{l}\text { Discrete } \\
\text { disturbance } \\
\text { factors }\end{array}$} & $\begin{array}{l}\text { Change to the } \\
\text { working } \\
\text { efficiency of } \\
\text { the human } \\
\text { workforce }\end{array}$ & $\begin{array}{l}\text { Accumulated disturbance affects the } \\
\text { execution of the production plan } \\
\text { and production quality }\end{array}$ & Type B & fuzzy \\
\hline & $\begin{array}{l}\text { Absence of } \\
\text { the human } \\
\text { workforce }\end{array}$ & $\begin{array}{l}\text { Affects the involved station, less } \\
\text { effect on the current scheduling plan }\end{array}$ & Type C & medium \\
\hline & $\begin{array}{l}\text { Operating } \\
\text { mistake }\end{array}$ & $\begin{array}{l}\text { If there is little scrap material, this } \\
\text { will only affect the processing task } \\
\text { of the current batch; if there is } \\
\text { much, it will affect the processing } \\
\text { task of all batches }\end{array}$ & Type B & fuzzy \\
\hline
\end{tabular}


These factors can be divided into four categories, according to their origin: the inherent disturbance factors; disturbance factors during production; disturbance factors caused by changes to an enterprise's external environment; and discrete disturbance factors $[17,18]$. Table I illustrates these disturbance factors.

In order to make the production system effectively respond to job shop disturbance factors, the 16 disturbance factors listed in Table I are divided, according to their effect on the production process and their disturbance degree, into three categories: type A, type B, and type C. Type A disturbance factors are events which, as long as they occur once, can seriously affect the production system. These factors obey discrete distribution, such as urgent customer orders, or temporary production plan change. Type B disturbance factors are similar to type A disturbance factors in terms of their source and characteristics (discrete distribution). Their disturbance degree is, however, less intense than those of type A. They are characterized by fuzziness. Reasonable countermeasures should therefore be formulated according to the actual situation. Type $\mathrm{C}$ disturbance factors refer to those which have little effect on the production system, but change frequently; belonging to continuous disturbance events.

\section{AN IMPROVED HYBRID RESCHEDULING STRATEGY}

\subsection{Disturbance degree judgment based on a fuzzy neural network}

Because type B disturbance factors are characterized by fuzziness, it is difficult to establish a suitable function to describe the relationship between type B factors and the output value of the disturbance degree. Therefore, a fuzzy neural network is used to evaluate the degree of the disturbance of production systems by type B disturbance factors. A fuzzy neural network, which has the advantages of both fuzzy mathematics and a neural network, is very suitable for solving fuzzy, nonlinear and classified problems and is therefore often used in decision support systems [19-21].

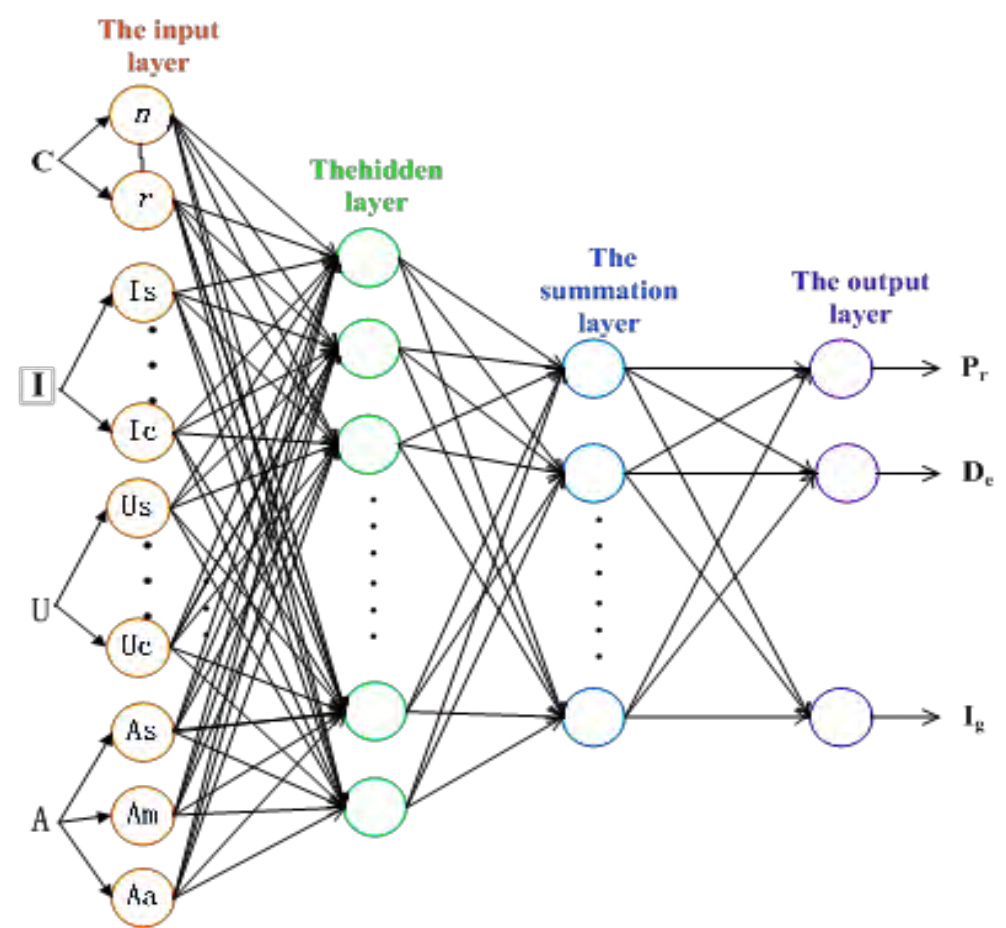

Figure 1: Structure chart of the probabilistic neural network.

In this paper, the fuzzy neural network is used to deal with type B disturbance factors and mainly includes two parts: fuzzy processing of the parameters, and training of the neural 
network. Fuzzy processing of the parameters is a pre-treatment of the original parameter data affecting the degree of disturbance of production systems. For instance, membership degree can be used to represent disturbance degree and can thus be normalized, as the input of a neural network. Neural network training is used to establish the nonlinear mapping relation between input parameters and disturbance degree. In this article, a probabilistic neural network is selected for the quantitative analysis of type B disturbance factors, due to its many advantages. These advantages include the simplicity of its training process, as well as its fast convergence speed, good fault tolerance, and arbitrary linear approximation. It is mainly composed of the input layer, the hidden layer, the summation layer and the output layer, as shown in Fig. 1.

According to Fig. 1, the input layer contains four parameters which affect how the effects of type B disturbance factors on the production system are judged. These parameters are, respectively: type of disturbance factors, C; occurrence strength of disturbance factors, I; urgent level of disturbance factors, $\mathrm{U}$; and cumulative intensity of disturbance factors, A. In the input layer, type B disturbance factors have two types: changes in the production environment, $n$, and resource scheduling failure, $r$. Except type parameter, all the other three parameters (I, U, and A) need fuzzy processing before input. At the same time, rescheduling requests $\left(P_{r}, D_{e}\right.$, and $\left.I_{g}\right)$ caused by type B disturbances are set as the output of the neural network. This reflects the degree of disturbance of the production system by type B disturbance factors. Among these requests, $P_{r}$ represents immediately executed rescheduling requests, $D_{e}$ represents delayed rescheduling requests, and $I_{g}$ represents negligible rescheduling requests. The output value is set as follows: the acceptance of the rescheduling request is 1 , while the refusal of the rescheduling request is 0 . The implementation of the fuzzy neural network method is not specified in this paper.

\subsection{Hybrid rescheduling strategy based on a fuzzy neural network}

In order to overcome the shortcomings of the existing rescheduling strategy, and after using a fuzzy neural network to analyze type B disturbance factors, an improved hybrid rescheduling strategy is proposed. The existing strategy was mainly improved in the following three aspects:

(1) A comprehensive analysis of the impact of various disturbance factors on the production system;

(2) Quantification of the disturbance degree of type B disturbance factors, and the reasonable selection of a processing method;

(3) The added constraint of a minimum rescheduling interval, which coordinates the response of the production system to a variety of disturbance factors and reduces rescheduling frequency.

The rescheduling strategy is as follows:

(1) For a disturbance factor generated in the manufacturing workshop, its type is first judged, its influence on the production system is then analysed, and finally the appropriate response mode is selected. If this disturbance factor is a type A factor, an immediate response is made, and if it is a type $\mathrm{C}$ factor, a periodic rescheduling strategy is used to respond to it. If the disturbance factor it is a type B factor, the disturbance degree of the system's factors is judged, according to quantitative analysis using the fuzzy neural network. Next, one of three rescheduling requests $\left(P_{r}, D_{e}\right.$, or $I_{g}$; caused by type B disturbance factors $)$ is obtained, as a production system response in the subsequent step. According to the above method, a rescheduling request set $T_{1}$ is obtained, where $T_{1}=\left\{t_{1 i} \mid t_{1 i}=t_{0}+\zeta\right\}, t_{0}$ is the starting time of the original schedule, and $\zeta$ is a period from the starting time to any rescheduling request time.

(2) Another set of rescheduling requests $T_{2}$ is obtained according to the periodic rescheduling strategy, where $T_{2}=\left\{t_{2 i} \mid t_{2 i}=t_{0}+t_{i}\right\}, t_{i}=i \cdot T_{0}$, and $i=1,2, \ldots, n . T_{0}$ is the rescheduling 
cycle, i.e. the time required for rescheduling, which is determined by staff according to actual conditions. Considering that $T_{1}$ and $T_{2}$ are generated according to different rescheduling strategies, the combination of these two leads to a large or small interval between the two adjacent rescheduling requests. The large interval situation is constrained by $T_{0}$, and the small interval situation by $\Delta T_{\min }$, which also avoids frequent rescheduling requests.

(3) Set $q_{i}(i=1,2, \ldots, n)$ is used as one of the rescheduling requests, and $S_{i}(i=1,2, \ldots, n)$ as the current scheduling plan.

a, if $q_{i} \in T_{2}$, rescheduling is started;

$\mathbf{b}$, if $q_{i} \in T_{1}$, the disturbance factor is a type A factor, and the production system is seriously affected, rescheduling is started immediately;

c, if $q_{i} \in T_{1}$ and the disturbance factor is a type B factor, the fuzzy neural network is used for quantitative analysis of the factor is impact on the production system. If a $P_{r}$-typed rescheduling request (i.e. $q_{i} \in P_{r}$ ) is obtained, rescheduling is started immediately. If a $D_{e^{-}}$ typed rescheduling request (i.e. $q_{i} \in D_{e}$ ) is obtained, $\Delta t$ (i.e. the interval between the starting time of the last rescheduling plan $S_{i-1}$ and the current rescheduling plan) is calculated. If $\Delta t>\Delta T_{\min }$, rescheduling is started immediately; if $\Delta t<\Delta T_{\min }, t_{1 i}$ is replaced with a new rescheduling request time, $t_{1 i}$ '. Where $t_{1 i}=t_{1 i}$ ' $\Delta t+\Delta T_{\min }$, rescheduling is started at the moment of $t_{1 i}$ '. If an $I_{g}$-typed rescheduling request (i.e. $q_{i} \in I_{g}$ ) is obtained, rescheduling is unnecessary, as the disturbance factor has little influence on production systems.

d, If $q_{i} \in T_{1}$, and the disturbance factor is a type $\mathrm{C}$ factor, it is processed according to the rescheduling request obtained by using the periodic rescheduling strategy.

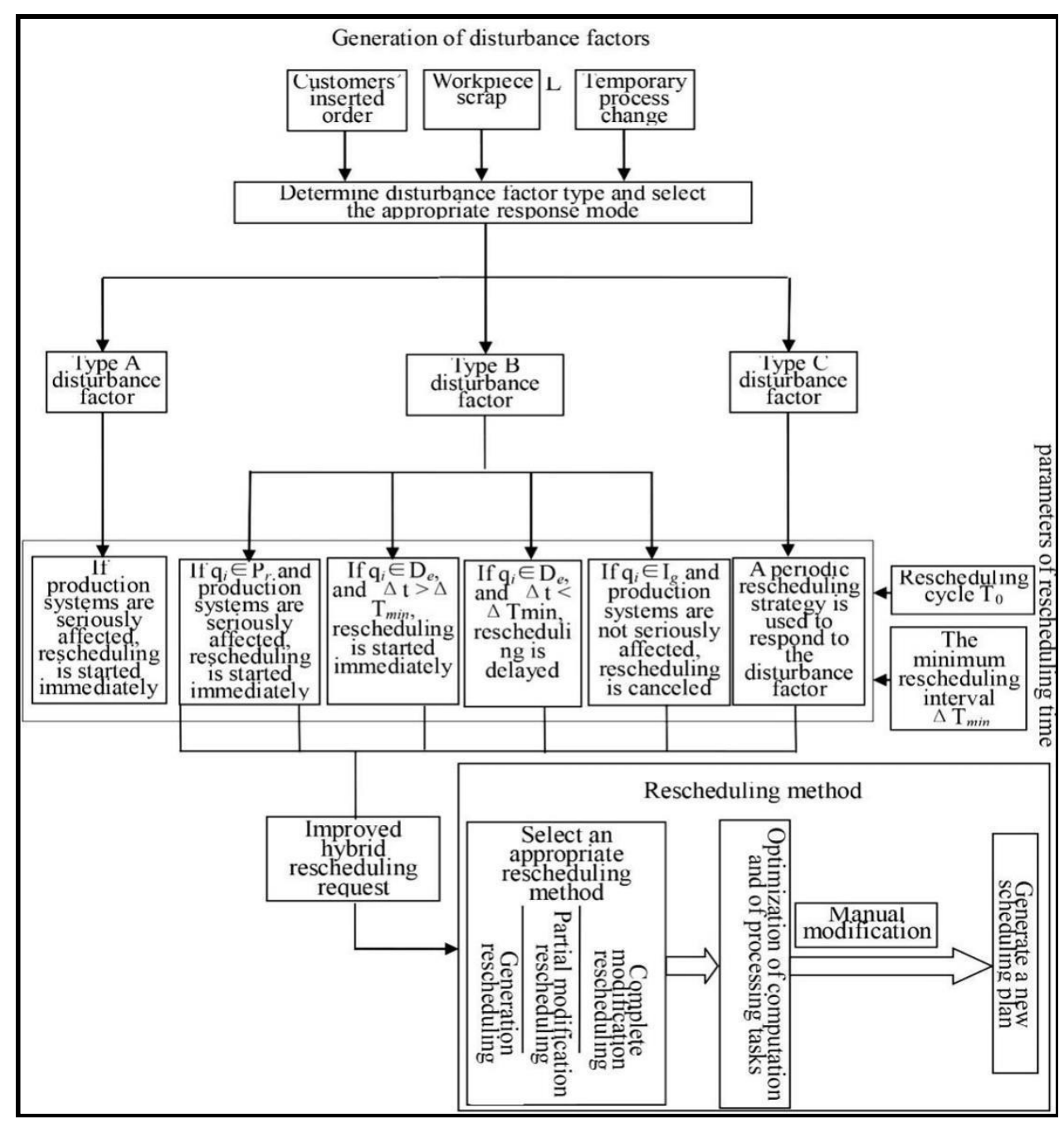

Figure 2: Flow chart of an improved hybrid rescheduling strategy. 
The flow chart of a rescheduling strategy is shown in Fig. 2. Using this method, not only can rescheduling be completed within the specified rescheduling cycle, but all kinds of disturbance factors produced in the manufacturing plant can also be effectively dealt with, especially those disturbance factors with fuzzy characteristics. At the same time, the strategy adds a minimum rescheduling interval constraint, which coordinates the relationship of the interval between periodic rescheduling requests and event driven rescheduling requests; effectively reducing rescheduling times.

\section{APPLICATION OF THE RESCHEDULING STRATEGY AND SIMULATION RESULTS}

A flexible job shop containing 6 sets of processing machines $\left(M_{1}, M_{2}, M_{3}, M_{4}, M_{5}\right.$, and $\left.M_{6}\right)$ and with five workpieces $\left(\mathrm{J}_{1}, \mathrm{~J}_{2}, \mathrm{~J}_{3}, \mathrm{~J}_{4}\right.$, and $\left.\mathrm{J}_{5}\right)$ to be processed, was selected as the object of study. The optional machines for each process and the time required to process workpieces in different machines are shown in Table II and Table III, respectively. When disturbance factors, such as a customer inserted order, a processing machine failure, or a processing time offset, were generated, they were dealt with using the rescheduling strategy and process proposed in this paper. The progress and the results were then analysed.

Table II: Optional machines for each process.

\begin{tabular}{|c|c|c|c|c|c|}
\hline \multirow{2}{*}{ Workpiece } & \multicolumn{5}{|c|}{ Optional machines for each process } \\
\cline { 2 - 6 } & process1 & process2 & process3 & process4 & process5 \\
\hline workpiece1 & $1,2,3$ & $2,4,5$ & $1,2,3$ & $1,4,5$ & $2,3,4,5,6$ \\
\hline workpiece2 & $1,3,5$ & $1,2,5$ & $3,5,6$ & $2,4,6$ & $1,2,3,5$ \\
\hline workpiece3 & 1,2 & $2,4,5$ & $3,5,6$ & $1,2,3,4,5$ & $1,2,5,6$ \\
\hline workpiece4 & $1,3,4$ & $2,4,6$ & $1,3,6$ & $1,3,4,5,6$ & $2,3,4,5$ \\
\hline workpiece5 & $1,2,3,4,5,6$ & $1,2,4,5$ & $1,3,6$ & $2,4,6$ & $1,3,4,5$ \\
\hline
\end{tabular}

Table III: The processing time of different workpieces.

\begin{tabular}{|c|c|c|c|c|c|}
\hline \multirow{2}{*}{ Workpiece } & \multicolumn{5}{|c|}{ Processing time (min) } \\
\cline { 2 - 6 } & process1 & process2 & process3 & process4 & process5 \\
\hline workpiece1 & $2,3,4$ & $3,2,4$ & $1,4,5$ & $4,3,5$ & $6,8,7,6,9$ \\
\hline workpiece2 & $3,5,2$ & $4,3,6$ & $4,7,11$ & $5,7,5$ & $4,5,7,5$ \\
\hline workpiece3 & 5,6 & $4,3,5$ & $13,9,12$ & $6,5,7,4,8$ & $8,6,7,8$ \\
\hline workpiece4 & $9,7,9$ & $6,4,5$ & $1,3,3$ & $6,9,7,5,4$ & $8,7,8,8$ \\
\hline workpiece5 & $4,3,7,9,3,6$ & $5,6,4,5$ & $6,4,3$ & $5,7,7$ & $7,8,7,8$ \\
\hline
\end{tabular}

(1) Generation of the initial scheduling scheme

According to the scale and computing complexity of the scheduling problem of the flexible job shop, the problem was solved using a dual layer encoding genetic algorithm. The parameters of this genetic algorithm were as follows: population size $P=100$, maximum number of iterations $M A X=400$, crossover probability $P_{c}=0.8$, mutation probability $P_{m}=0.1$, and generation gap 0.1. After iterative calculation, the initial scheduling scheme was obtained, with the maximum completion time of $27 \mathrm{~min}$. The processing procedures are shown in Fig. 3.

(2) Optimization simulation of the rescheduling scheme

If three different kinds of disturbance factors are all generated in the course of the execution of the scheduling scheme, they are dealt with using the previously proposed fuzzy neural network. The disturbance degree of the production systems and the corresponding rescheduling requests were obtained, as shown in Table IV. 


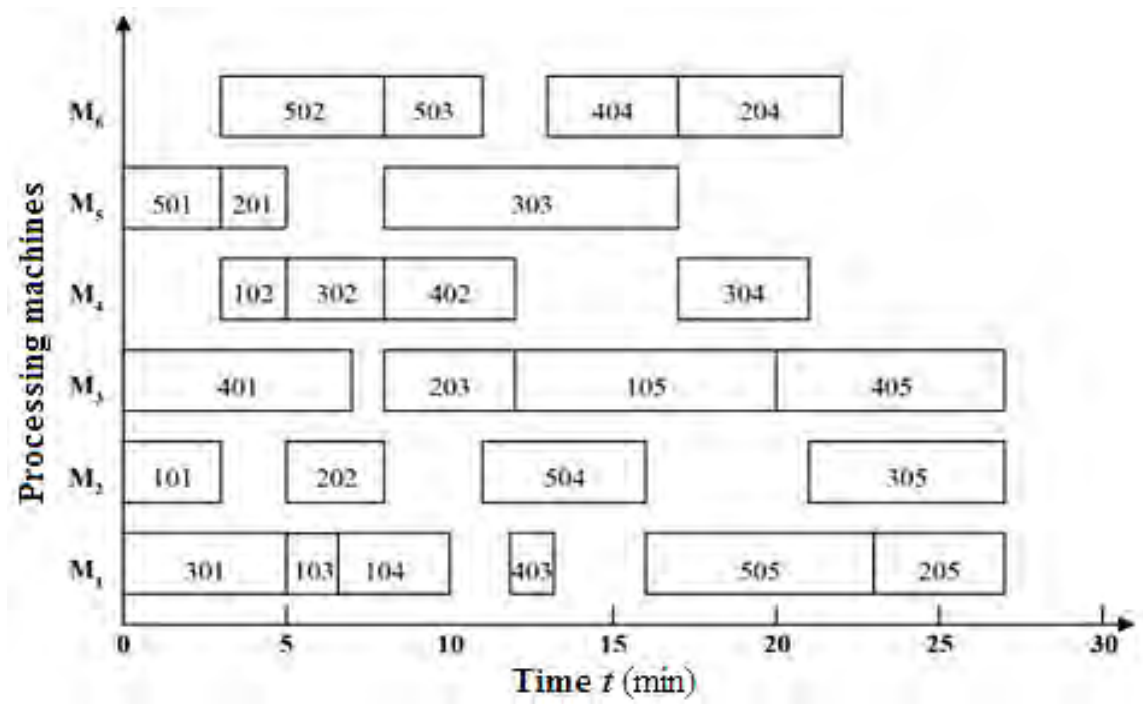

Figure 3: The initial rescheduling plan.

Table IV: Classification of disturbance factors and output of the neural network.

\begin{tabular}{|c|l|c|c|c|c|}
\hline \multirow{2}{*}{ No. } & \multirow{2}{*}{ Disturbance factors } & \multirow{2}{*}{ Disturbance mode } & \multicolumn{3}{|c|}{ Output of the neural network } \\
\cline { 4 - 6 } & & & $\boldsymbol{P}_{\boldsymbol{r}}$ & $\boldsymbol{D}_{\boldsymbol{e}}$ & $\boldsymbol{I}_{\boldsymbol{g}}$ \\
\hline 1 & Customer's inserted order & A-typed & 1 & 0 & 0 \\
\hline 2 & Processing machine failure & B-typed & 1 & 0 & 0 \\
\hline 3 & Processing time offset & C-typed & 0 & 1 & 0 \\
\hline
\end{tabular}

(1) Customer's inserted order

When type A disturbance factors, like a customer's inserted order, emerge, the new production task is added to the original production scheduling scheme, according to the new order index (such as the date of delivery, or the number of workpieces). Thus, a new scheduling scheme is generated. Disturbance factors of this kind therefore have a great influence on production systems. When dealing with this kind of disturbance factor the generative rescheduling method can be used. Priority is thereby given to tasks in their inserted order, and the other tasks affected by the customer emergency order are then reorganized.

When the initial scheduling scheme was executed for 13 minutes, a notice was received of the need for processing workpieces $\left(\mathrm{J}_{6}, \mathrm{~J}_{7}\right.$, and $\left.\mathrm{J}_{8}\right)$. Optional machines for each process and the times required to process workpieces in different machines are shown in Table $\mathrm{V}$ and Table VI, where * represents the absence of any process.

Table V: Optional machines for each process of inserted workpieces.

\begin{tabular}{|c|c|c|c|c|c|}
\hline \multirow{2}{*}{ Workpiece } & \multicolumn{5}{|c|}{ Optional machines for each process } \\
\cline { 2 - 6 } & process1 & process2 & process3 & process4 & process5 \\
\hline workpiece6 & $2,3,4,5$ & $1,3,5,6$ & $2,3,5$ & $1,3,4,5$ & $1,4,5,6$ \\
\hline workpiece7 & $1,2,3,5$ & $2,3,4,5$ & $1,3,5,6$ & $*$ & $*$ \\
\hline workpiece8 & $2,4,6$ & $1,2,4,5$ & $2,4,5,6$ & $1,3,4$ & $*$ \\
\hline
\end{tabular}

Table VI: The processing times of different inserted workpieces.

\begin{tabular}{|c|c|c|c|c|c|}
\hline \multirow{2}{*}{ Workpiece } & \multicolumn{5}{|c|}{ Processing time (min) } \\
\cline { 2 - 6 } & process1 & process2 & process3 & process4 & process5 \\
\hline workpiece6 & $5,6,4,7$ & $5,6,4,7$ & $4,5,5$ & $4,7,6,5$ & $5,7,6,7$ \\
\hline workpiece7 & $3,4,5,5$ & $5,4,5,6$ & $6,5,6,9$ & $*$ & $*$ \\
\hline workpiece8 & $6,7,5$ & $5,4,3,5$ & $5,7,9,12$ & $6,7,4$ & $*$ \\
\hline
\end{tabular}


The following tasks were then reorganized using the generative rescheduling method, with priority given to tasks in their inserted order. According to the scheduling rules, a processed workpiece should continue to be processed, until the corresponding process is finished (such as the third process of the workpiece $\mathrm{J}_{3}$, the fifth process of the workpiece $\mathrm{J}_{1}$ and the fourth processes of the workpiece $J_{5}$ in Fig. 4). The processing of workpieces after 13 minutes is optimized using the double layer encoding genetic algorithm; obtaining a rescheduling scheme with a maximum completion time of 35 minutes. From the following figure, it can be seen that processing priority was given to the three urgent workpieces. The remaining operations of processing $\mathrm{J}_{1}, \mathrm{~J}_{2}, \mathrm{~J}_{3}, \mathrm{~J}_{4}$, and $\mathrm{J}_{5}$ were carried out upon completion of the inserted order. Workpiece $J_{1}$ in the fifth process, workpiece $J_{3}$ in the fourth process, and workpiece $J_{3}$ in the fifth process were also processed not in their original machines, but in new ones.

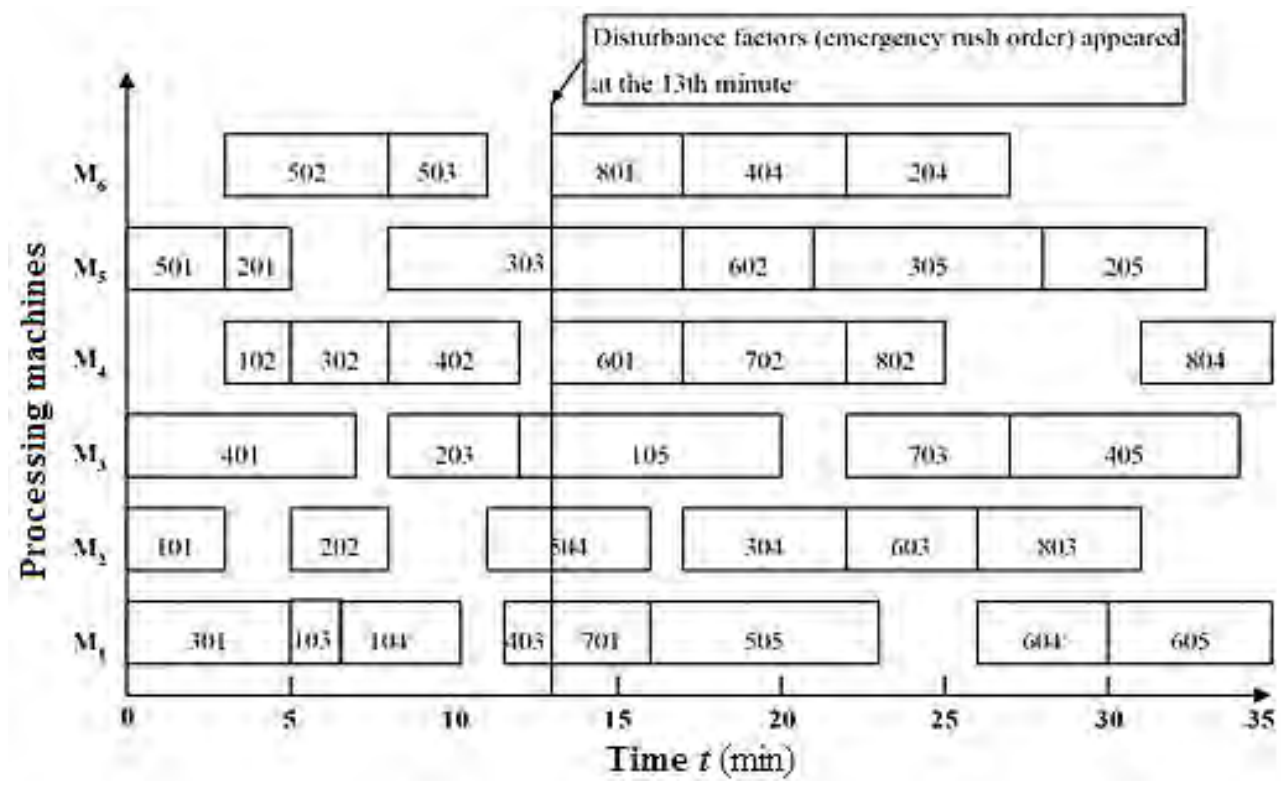

Figure 4: The rescheduling plan after reception of a customer's inserted order.

(2) Equipment failure

When a machine breaks down, a fuzzy neural network algorithm is used first. This determines the influence of the disturbance factors on the production system and obtains the $P_{r}, D_{e}$ and $I_{g}$ rescheduling requests, as the response mode of the production system. Next, according to the results of the preceding step, the appropriate rescheduling method for processing is selected. In the flexible job shop, each process of a workpiece can be completed on different machines. The partial modification rescheduling method can therefore be used to respond to disturbances. The partial modification rescheduling method is specified as follows: if there is a machine in the same category as the breakdown one in the workshop, the remaining work to be done in the breakdown machine can be transferred to this other machine; if there is no machine in the same category as the breakdown one in the workshop, it is necessary to analyse whether the affected process has an alternative process. If this is the case, the alternative is used, if not, the remaining work to be done in the breakdown machine will be transferred to other machines.

In this paper, it is assumed that, at the $13^{\text {th }}$ minute, processing machine $\mathrm{M}_{6}$ in the initial scheduling scheme broke down and could not be repaired within a short period. At the same time, there were no processing machines in the same category as $\mathbf{M}_{6}$. The remaining processing tasks therefore needed to be completed on a newly-selected machine, and the processing sequence needed to be rearranged. According to Fig. 3, an initial schedule Gantt chart, when machine $\mathrm{M}_{6}$ broke down, some processes were directly affected, including 
workpiece $\mathrm{J}_{4}$ in the fourth process and workpiece $\mathrm{J}_{2}$ in the fourth process. Others were indirectly affected, including workpiece $J_{3}$ in the fifth process. In the initial scheduling scheme, the priority of the workpieces given to $\mathrm{J}_{1}, \mathrm{~J}_{2}, \mathrm{~J}_{3}, \mathrm{~J}_{4}$, and $\mathrm{J}_{5}$ was: $2,4,3,5$, and 2 . To perform quantitative analysis of the disturbance factor using a fuzzy neural network, fuzzy processing of the parameters is first carried out:

a, The type of disturbance factor $C$ is determined.

Equipment failure is one kind of resource scheduling failure $r$.

b, The intensity of the disturbance factor $I$ is calculated.

The number of relative processes affected by the disturbance $n_{r}$ is:

$$
n_{r}=\frac{n_{a}}{N}=\frac{3}{25}=0.12
$$

The membership function $R(x)$ is used to calculate the membership degree of subsets $I_{s}$, $I_{w}, I_{m}$, and $I_{c}$. The results are as follows: $I_{s}=0.6, I_{w}=0.69, I_{m}=0.02$, and $I_{c}=0$.

c, The emergency degree of the disturbance factor $U$ is calculated.

The relative priority of the working procedure affected by the disturbance factor $p_{m}$ is:

$$
p_{m}=\frac{\sum_{i=1}^{n_{a}} p_{i}}{n_{a}} / P_{\max }=\frac{(5+4+3)}{3} / 5=0.8
$$

The membership function $R(x)$ is used to calculate the membership degree of subsets $U_{s}$, $U_{w}, U_{m}, U_{a}$ and $U_{c}$. The results are as follows: $U_{s}=0, U_{w}=0, U_{m}=0.11, U_{a}=0.32$ and $U_{c}=0$.

d, The cumulative intensity of the disturbance factor $A$ is calculated.

The relative amount of the accumulated working process affected by the disturbance factor $n_{a r}$ is:

$$
n_{a r}=\frac{n_{a a}}{N}=\frac{10}{25}=0.4
$$

The membership function $R(x)$ is used to calculate the membership degree of subsets $A_{w}$, $A_{m}$, and $A_{a}$. The results are as follows: $A_{s}=0.01, A_{w}=0.72$, and $A_{a}=0$.

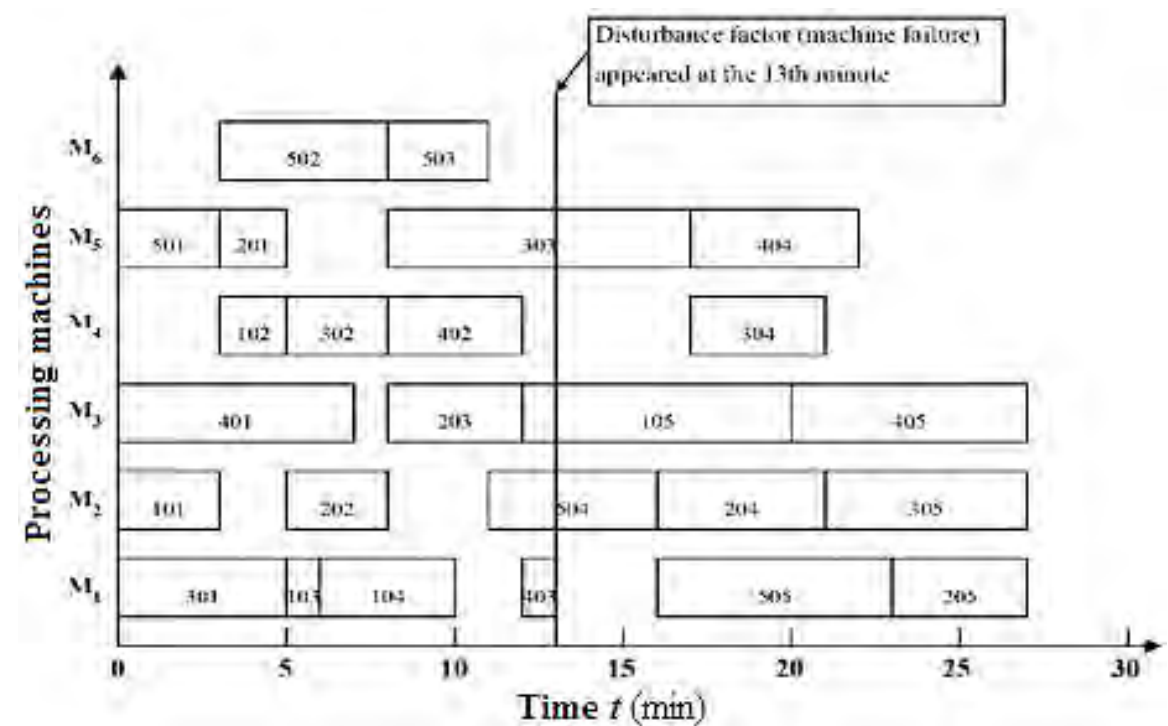

Figure 5: The rescheduling plan after the breakdown of processing machine M6. 
The above calculation of membership values was used as input to the fuzzy neural network algorithm. $A$ simulation was then conducted using MATLAB. The rescheduling requests $P_{r}, D_{e}$ and $I_{g}$ caused by type B disturbance factors are as follows: $P_{r}=1, D_{e}=0, I_{g}=0$.

As can be seen from the result of the rescheduling request, the production workshop needed to be rescheduled immediately. According to the number and characteristics of the affected processes, a partial modification method was used to generate a rescheduling scheme. By using the combination of an intelligent optimization algorithm and manual scheduling, a scheduling scheme with the maximum completion time of 27 minutes was obtained, as shown in Fig. 5. It can be seen from this figure that the fourth process of workpiece $J_{2}$ and the fourth process of workpiece $\mathrm{J}_{4}$ were transferred to machine $\mathrm{M}_{2}$ and machine $\mathrm{M}_{5}$, respectively.

(3) Processing time offset

According to data analysis of the production system, after the customer inserted order was processed, a kind of type $\mathrm{C}$ disturbance factor (processing time offset) was generated. This kind of disturbance had little effect on the production system and could be eliminated using a periodic rescheduling strategy. In this case, the data reflecting the processing time offset are as follows: due to a toggle clamping fixture, workpiece $J_{5}$ on machine $M_{1}$ had a 10 minute delay in processing time; because of a temporary delivery date change, the fifth process of workpiece $\mathrm{J}_{4}$ on machine $\mathrm{M}_{3}$ was finished before the completion of the third process of workpiece $\mathrm{J}_{7}$; due to a material transportation problem, the fourth process of workpiece $\mathrm{J}_{2}$ on machine $\mathrm{M}_{6}$ needed to be postponed by 5 minutes before it could then be processed.

According to the disturbance degree of these disturbance factors on the production system, as well as to the complexity of the scheduling problem, at the $21^{\text {st }}$ minute, when the rescheduling cycle arrived, the complete modification rescheduling method was used. This was combined with manual adjustment and an intelligent optimization algorithm to optimize the subsequent processes. A rescheduling scheme with the maximum completion time of 37 minutes was obtained. From Fig. 6, it can be seen that the fourth process of workpiece $\mathrm{J}_{6}$ (604) on machine $\mathrm{M}_{1}$, the fifth process of workpiece $\mathrm{J}_{6}(605)$ and the fourth process of workpiece $\mathrm{J}_{2}(204)$ on machine $\mathrm{M}_{5}$ were completed on new machines.

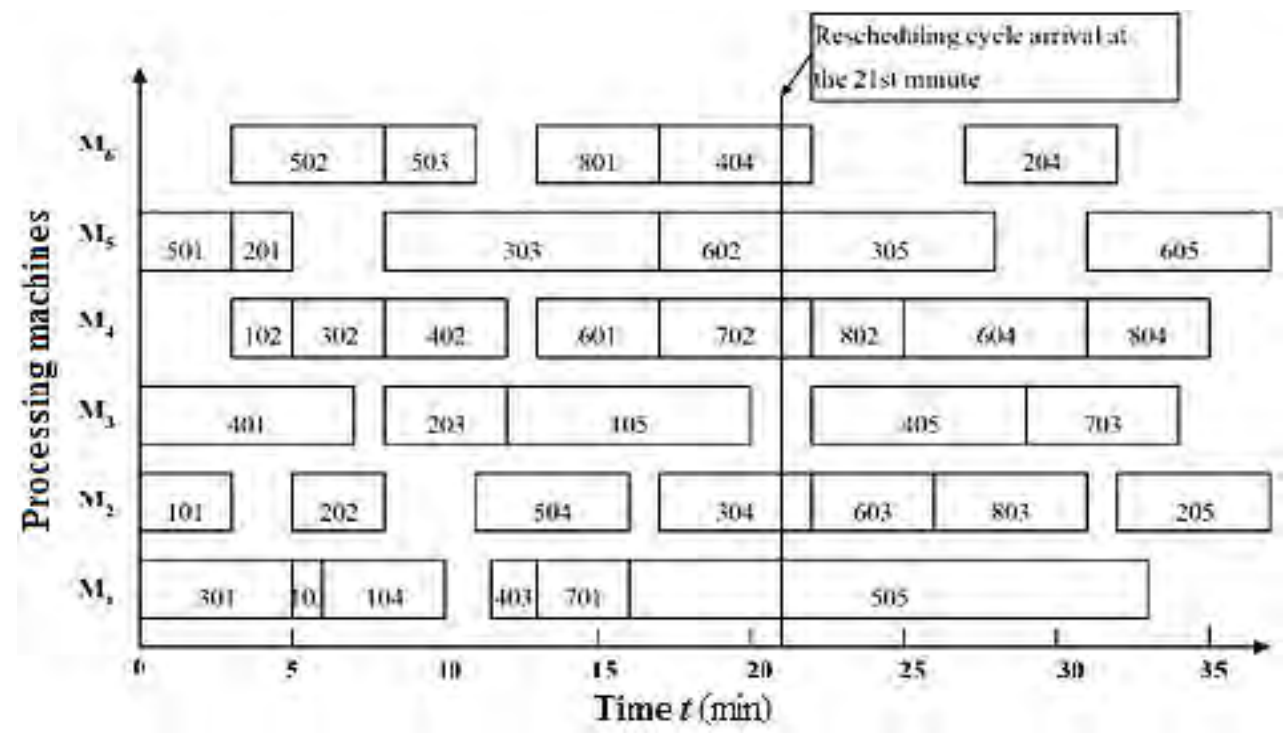

Figure 6: The rescheduling plan after the generation of a processing time offset.

The simulation results show that the adaptive rescheduling process proposed in this paper can effectively solve rescheduling problems of flexible job shops with different kinds of disturbance factors. An appropriate scheduling strategy and corresponding rescheduling method, which are chosen according to the impact of different kinds of disturbance factors on 
the production system, can reduce rescheduling times, and the corresponding cost of calculation.

\section{CONCLUSION}

In order to eliminate the shortcomings of the existing rescheduling strategy, an improved hybrid rescheduling strategy was proposed for manufacturing firms, after using a fuzzy neural network to analyse type B disturbance factors. On the basis of this, an adaptive rescheduling process for a manufacturing system was constructed and an improved hybrid rescheduling strategy was proposed. The effectiveness of this improved strategy was illustrated by a case study of practical manufacturing. The simulation results show that this strategy can effectively eliminate the influence of various disturbance factors in a production system and can also reduce rescheduling times and maintain the stability of manufacturing systems. This provides a practical and effective solution to market change for firms adopting an MTO mode.

\section{ACKNOWLEDGEMENT}

This research is funded by The Fundamental and Advanced Research Projects of Chongqing city in China (CSTC2013jcyjA0564).

\section{REFERENCES}

[1] Li, H.-T.; Womer, K. (2012). Optimizing the supply chain configuration for make-to-order manufacturing, European Journal of Operational Research, Vol. 221, No. 1, 118-128, doi:10.1016/j.ejor.2012.03.025

[2] Zhang, Z. G.; Kim, I.; Springer, M.; Cai, G. G.; Yu, Y.-G. (2013). Dynamic pooling of make-tostock and make-to-order operations, International Journal of Production Economics, Vol. 144, No. 1, 44-56, doi:10.1016/j.ijpe.2013.01.012

[3] Yu, C.; Ji, Y.; Qi, G.; Gu, X.; Tao, L. (2015). Group-based production scheduling for make-toorder production, Journal of Intelligent Manufacturing, Vol. 26, No. 3, 585-600, doi: $10.1007 / \mathrm{s} 10845-013-0817-\mathrm{z}$

[4] Qiao, F.; Li, L.; Wang, Z.-T.; Guo, C.-Z. (2007). Improved hybrid rescheduling strategy for semiconductor manufacturing, Computer Integrated Manufacturing Systems, Vol. 13, No. 3, 558562

[5] Dong, Y.-H.; Jang, J. (2012). Production rescheduling for machine breakdown at a job shop, International Journal of Production Research, Vol. 50, No. 10, 2681-2691, doi:10.1080/ 00207543.2011.579637

[6] ElMaraghy, H. A.; ElMekkawy, T. Y. (2002). Deadlock-free rescheduling in flexible manufacturing systems, CIRP Annals - Manufacturing Technology, Vol. 51, No. 1, 371-374, doi:10.1016/S0007-8506(07)61539-X

[7] Zakaria, Z.; Petrovic, S. (2012). Genetic algorithms for match-up rescheduling of the flexible manufacturing systems, Computers \& Industrial Engineering, Vol. 62, No. 2, 670-686, doi: $10.1016 /$ j.cie.2011.12.001

[8] Chen, K. J.; Ji, P. (2007). A genetic algorithm for dynamic advanced planning and scheduling (DAPS) with a frozen interval, Expert Systems with Applications, Vol. 33, No. 4, 1004-1010, doi:10.1016/j.eswa.2006.08.025

[9] Pfund, M. E. (2002). Evaluation of uncertainty on scheduling algorithms in printed wiring board manufacturing (PhD Dissertation), Arizona State University, Tempe

[10] Guo, B.; Nonaka, Y. (1999). Rescheduling and optimization of schedules considering machine failures, International Journal of Production Economics, Vol. 60-61, 503-513, doi:10.1016/ $\underline{\mathrm{S} 0925-5273(98) 00155-8}$

[11] Akturk, M. S.; Gorgulu, E. (1999). Match-up scheduling under a machine breakdown, European Journal of Operational Research, Vol. 112, No. 1, 81-97, doi:10.1016/S0377-2217(97)00396-2 
[12] Li, Y.-W.; Gujrati, P.; Lan, Z.-L.; Sun, X.-H. (2007). Fault-driven rescheduling for improving system-level fault resilience, Proceedings of the International Conference on Parallel Processing, Session 4B, paper 39, doi:10.1109/ICPP.2007.42

[13] Mendez, C. A.; Cerda, J. (2003). Dynamic scheduling in multiproduct batch plants, Computers \& Chemical Engineering, Vol. 27, No. 8-9, 1247-1259, doi:10.1016/S0098-1354(03)00050-4

[14] Church, L. K.; Uzsoy, R. (1992). Analysis of periodic and event driven rescheduling policies in dynamic shops, International Journal of Computer Integrated Manufacturing, Vol. 5, No. 3, 153163, doi:10.1080/09511929208944524

[15] Rangsaritratsamee, R.; Ferrell Jr., W. G.; Kurz, M. B. (2004). Dynamic rescheduling that simultaneously considers efficiency and stability, Computers \& Industrial Engineering, Vol. 46, No. 1, 1-15, doi: 10.1016/j.cie.2003.09.007

[16] Liu, G.-B. (2013). Dynamic schedule method based on improved rolling time domain optimization strategy, Chinese Journal of Mechanical Engineering, Vol. 49, No. 14, 182-190, doi:10.3901/JME.2013.14.182

[17] Honkomp, S. J.; Mockus, L.; Reklaitis, G. V. (1999). A framework for schedule evaluation with processing uncertainty, Computers \& Chemical Engineering, Vol. 23, No. 4-5, 595-609, doi:10.1016/S0098-1354(98)00296-8

[18] Katragjini, K.; Vallada, E.; Ruiz, R. (2013). Flow shop rescheduling under different types of disruption, International Journal of Production Research, Vol. 51, No. 3, 780-797, doi: $10.1080 / 00207543.2012 .666856$

[19] Rubaai, A.; Young, P. (2011). EKF-based PI-/PD-like fuzzy-neural-network controller for brushless drives, IEEE Transactions on Industry Applications, Vol. 47, No. 6, 2391-2401, doi:10.1109/TIA.2011.2168799

[20] Otadi, M. (2014). Fully fuzzy polynomial regression with fuzzy neural networks, Neurocomputing, Vol. 142, 486-493, doi:10.1016/j.neucom.2014.03.048

[21] Kuo, R. J.; Tseng, W. L.; Tien, F. C.; Liao, T. W. (2012). Application of an artificial immune system-based fuzzy neural network to a RFID-based positioning system, Computers \& Industrial Engineering, Vol. 63, No. 4, 943-956, doi:10.1016/j.cie.2012.06.006 